\title{
Implications of the first neutral current data from SNO for Solar Neutrino Oscillation
}

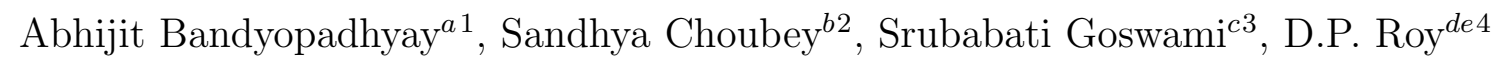

${ }^{a}$ Saha Institute of Nuclear Physics, Bidhannagar, Kolkata 700 064, INDIA

${ }^{b}$ Department of Physics and Astronomy, University of Southampton, Highfield, Southampton S017 1BJ, UK

$c$ Harish-Chandra Research Institute, Chhatnag Road, Jhusi, Allahabad - 211-019, INDIA

${ }^{d}$ Tata Institute of Fundamental Research, Homi Bhabha Road, Mumbai 400 005, INDIA

${ }^{e}$ Physics Department, University of California, Riverside, Ca 92521,USA

\begin{abstract}
We perform model independent and model dependent analyses of solar neutrino data including the neutral current event rate from SNO. The inclusion of the first SNO $\mathrm{NC}$ data in the model independent analysis determines the allowed ranges of ${ }^{8} B$ flux normalisation and the $\nu_{e}$ survival probability more precisely than what was possible from the SK and SNO CC combination. We perform global $\nu_{e}-\nu_{\text {active }}$ oscillation analyses of solar neutrino data using the NC rate instead of the SSM prediction for the ${ }^{8} B$ flux, in view of the large uncertainty in the latter. The LMA gives the best solution, while the LOW solution is allowed only at the $3 \sigma$ level.
\end{abstract}

\footnotetext{
${ }^{1}$ e-mail: abhi@theory.saha.ernet.in

${ }^{2}$ email: sandhya@hep.phys.soton.ac.uk

${ }^{3}$ e-mail: sruba@mri.ernet.in

${ }^{4}$ e-mail:dproy@theory.tifr.res.in
} 
The neutral current results from the Sudbury Neutrino Observatory measures for the first time the total flux of ${ }^{8} B$ neutrinos coming from the Sun [1]. In a recent paper [2] we had examined the role of the anticipated NC data from SNO in enhancing our understanding of the solar neutrino problem. The SNO NC rate can be expressed in terms of SNO CC and SK elastic scattering rates as [3]

$$
R_{S N O}^{N C}=R_{S N O}^{C C}+\left(R_{S K}^{e l}-R_{S N O}^{C C}\right) / r
$$

where $r=\sigma_{\nu_{\mu}, \tau}^{N C} / \sigma_{\nu_{e}}^{C C+N C} \simeq 0.157$ for a threshold energy of $5 \mathrm{MeV}$ (including the detector resolutions and the radiative corrections to $\nu-e$ scattering cross-sections). All the rates are defined with respect to the BBP2000 Standard Solar Model (SSM) 团. We showed in [2] that because SNO has a greater sensitivity to the NC scattering rate as compared to SK, the SNO NC measurement will be more precise and hence incorporation of this can be more predictive than the SNO CC and SK combination. We took three representative NC rates $-R_{N C}^{S N O}=0.8,1.0$ and $1.2( \pm 0.08)$ and showed that

1. For a general transition of $\nu_{e}$ into a mixture of active and sterile neutrinos the size of the sterile component can be better constrained than before.

2. For transition to a purely active neutrino the ${ }^{8} B$ neutrino flux normalisation and the survival probability $P_{e e}$ are determined more precisely.

3. We had also performed global two flavour oscillation analysis of the solar neutrino data for the $\nu_{e}-\nu_{\text {active }}$ case, where instead of $R_{S K}$ and $R_{S N O}^{C C}$ we used the quantities $R_{S K}^{e l} / R_{S N O}^{N C}$ and $R_{S N O}^{C C} / R_{S N O}^{N C}$. These ratios are independent of the ${ }^{8} B$ flux normalisation and hence of the SSM uncertainty. We showed that use of these ratios can result in drastic reduction of the allowed parameter regions specially in the LOW-QVO area depending on the value of the NC rate.

We now have the actual experimental result

$$
R_{S N O}^{N C}=1.01 \pm 0.12
$$

while eq. (1) gives $1.05 \pm 0.15$. Thus in 306 live days (577 days) the SNO NC measurement has achieved a precision, which is already better than that obtained from the SK and SNO CC combination. This paper follows closely the analysis that we have done in [2] but incorporating the actual data. In addition we also perform an alternative global analysis for $\nu_{e}-\nu_{\text {active }}$ oscillation by letting the ${ }^{8} B$ normalisation factor $f_{B}$ vary freely, where the inclusion of $R_{S N O}^{N C}\left(=f_{B}\right)$ in the fit serves to control this parameter. As we shall see below the two methods of global analysis give very similar results.

In section 1 we discuss the constraints on the electron neutrino survival probability, the ${ }^{8} B$ normalisation factor $f_{B}$ and the fraction of sterile component without assuming any particular model for the probabilities. In section 2 we perform the global analyses assuming two flavour $\nu_{e}-\nu_{\text {active }}$ oscillation. 


\section{Model Independent Analysis}

For the general case of $\nu_{e}$ transition into a combination of $\nu_{\text {active }}\left(\nu_{a}\right)$ and $\nu_{\text {sterile }}\left(\nu_{s}\right)$ states one can write the SK, SNO CC and SNO NC rates as

$$
\begin{aligned}
R_{S K}^{e l} & =f_{B} P_{e e}+f_{B} r P_{e a} \\
R_{S N O}^{C C} & =f_{B} P_{e e} \\
R_{S N O}^{N C} & =f_{B}\left(P_{e e}+P_{e a}\right)
\end{aligned}
$$

where $P_{e e}$ and $P_{e a}$ denote the probabilities folded with the detector response function [5] and averaged over energy. To extract a model independent bound on $P_{e e}$ one has to ensure an equality of the response functions which amounts to slight adjustment of the SK threshold energy and the rate [5, 6]. Our approach is slightly different. We treat $P_{e e}$ to be effectively energy independent. The SK spectrum data indicates a flat probability down to $5 \mathrm{MeV}$ [7]. This is corroborated by SNO [8, 9] which now has a threshold of $5 \mathrm{MeV}$ for kinetic energy of the observed electron. Hence we consider this assumption as justified and expect the results to be insensitive to the differences in the response functions. It should be noted here that in cotrast to the SNO CC events their NC events correspond to a neutrino energy threshold of $2.2 \mathrm{MeV}$. However it is clear from eq. (5) that for a $\nu_{e}$ to $\nu_{a}$ transition there is no reason to expect any energy dependence in $R_{S N O}^{N C}$. On the other hand for the general case of $\nu_{e}$ transition into a combination of $\nu_{a}$ and $\nu_{s}$ our approach effectively assumes $P_{e s}$ to be energy independent down to $2.2 \mathrm{MeV}$. A comparison of the current values $R_{S N O}^{C C}$ with $R_{S N O}^{N C}$ is shown in fig. 1. It constitutes a $5.3 \sigma$ signal for transition to a state containing an active neutrino component or alternatively a $5.3 \sigma$ signal against a pure sterile solution.

Next we consider the general case where $\nu_{e}$ goes to a mixed state $=\nu_{a} \sin \alpha+\nu_{s} \cos \alpha$. Then one can write $P_{e a}=\sin ^{2} \alpha\left(1-P_{e e}\right)$. Substituting this in the equations (3) and (5) and eliminating $P_{e e}$ using equation (雨) one gets the following set of equations for $f_{B}$ and $\sin ^{2} \alpha[2$

$$
\begin{aligned}
& \sin ^{2} \alpha\left(f_{B}-R_{S N O}^{C C}\right)=\left(R_{S K}^{e l}-R_{S N C}^{C C}\right) / r \\
& \sin ^{2} \alpha\left(f_{B}-R_{S N O}^{C C}\right)=R_{S N O}^{N C}-R_{S N O}^{C C}
\end{aligned}
$$

We treat $\sin ^{2} \alpha$ as a model parameter. And for different input values of $\sin ^{2} \alpha$ we determine the central value and the $1 \sigma$ and $2 \sigma$ ranges of $f_{B}$ by taking a weighted average of the equations (6) and (7). The corresponding curves are presented in fig. 2. Combining the 2 $\sigma$ lower limit of $f_{B}$ from this fit with the $2 \sigma$ upper limit from the SSM (vertical lines) gives a lower limit of $\sin ^{2} \alpha>0.45$ i.e. the probability of the active component is $>45 \%$. Note that there is no upper limit on this quantity - i.e. the data is perfectly compatible with $\nu_{e}$ transition into purely active neutrinos.

Assuming transition into purely active neutrinos $\left(P_{e a}=1-P_{e e}\right)$ we show in fig. 3 the $1 \sigma$ and $2 \sigma$ contours in the $f_{B}-P_{e e}$ plane from the combinations SK+SNOCC and $\mathrm{SK}+\mathrm{SNOCC}+\mathrm{SNONC}$. The inclusion of the NC rate narrows down the ranges of $f_{B}$ and $P_{e e}$. The error in $f_{B}$ after the inclusion of $\mathrm{NC}$ data is about half the size of the corresponding error from SSM as is seen from fig. 3 . 


\section{Model dependent analysis}

In this section we present the results of our $\chi^{2}$ analysis of solar neutrino rates and SK spectrum data in the framework of two flavour oscillation of $\nu_{e}$ to an active flavour. We use the standard techniques described in our earlier papers 10, 11 excepting for the fact that instead of the quantities $R_{S K}^{e l}$ and $R_{S N O}^{C C}$ we now fit the ratios $R_{S K}^{e l} / R_{S N O}^{N C}$ and $R_{S N O}^{C C} / R_{S N O}^{N C}$. The ${ }^{8} B$ flux normalisation gets cancelled from these ratios and the analysis becomes independent of the large (16-20\%) SSM uncertainty associated with this. We include in our global analyses the 1496 day SK zenith angle spectra [15]. Since we use both SK rate and SK spectrum data we keep a free normalisation factor for the SK spectrum. This amounts to taking the information on total rates from the SK rates data and the information of the spectral shape from the SK spectrum data. The SNO CC and NC rates have a large anticorrelation. We have taken into account this correlation between the measured SNO rates in our global analyses. Further details of this fitting method can be found in [2]. In Table 2 we present the best-fit parameters, $\chi_{\min }^{2}$ and goodness of fit (GOF). The best-fit comes in the $\mathrm{HIGH}(\mathrm{LMA})$ region as before [11, 12]. However as is seen from fig. 4a the incorporation of the NC data narrows down the allowed regions, and in particular the LOW region becomes much smaller.

We have also performed an alternative $\chi^{2}$ fit to the rates of Table 1 [1, 0, 13, 14] along with the 1496 day SK spectra [15], keeping $f_{B}$ as a free parameter. Even though we allow $f_{B}$ to vary freely the $\mathrm{NC}$ data serves to control $f_{B}$ within a range determined by its error. As we see from Table 2 and fig. $4 \mathrm{~b}$ the results of this fit are very similar to the previous case. The best fit comes from the HIGH(LMA) region, while no allowed region is obtained for the LOW solution at the 99\% CL level. Maximal mixing is seen to be disallowed at the $3 \sigma$ level. To illustrate the impact of the NC rate on the oscillation solutions we have repeated the free $f_{B}$ fit without this rate. The results are shown in fig 4c. Evidently the $\mathrm{NC}$ data plays a pivotal role in constraining the oscillation solutions, particularly in the LOW/QVO region, which is allowed only at the $3 \sigma$ level. It puts an upper bound on the $\Delta m^{2}$ in the LMA region and rules out maximal mixing.

\section{Summary and Conclusions}

The first SNO NC data constitutes a $5.3 \sigma$ signal for transition into a state containing an active neutrino component. The inclusion of this data puts much tighter constraints on $f_{B}$ and $P_{e e}$ from a model independent analysis involving active neutrinos as compared to the $\mathrm{SNO} \mathrm{CC} / \mathrm{SK}$ combination. In this paper we have discussed two useful strategies, of incorporating the NC data in the global $\chi^{2}$ analysis of rates and spectrum data, by which one can avoid the large ${ }^{8} B$ flux uncertainty from the SSM.

- We fit the ratios of the SK elastic and SNO CC rates w.r.t the NC rate, from which the $f_{B}$ cancels out.

- We fit the rates by keeping $f_{B}$ as a free parameter, where the inclusion of the SNO NC rate $\left(=f_{B}\right)$ serves to control this parameter.

Both the analyses give very similar results. They clearly favour the HIGH(LMA) solution, while a limited region of the LOW solution is also acceptable at the $3 \sigma$ level. The 
maximal mixing solution is disfavoured at the $3 \sigma$ level. As more data accumulate one expects a substantial reduction in the error bar of the SNO NC rate, resulting in further tightening of the allowed regions of neutrino mass and mixing.

Note Added: The paper [16] appeared on the net after completion of our work. In the region of overlap our results agree with theirs as well as with the updated version of [17]. It may be added here that the SNO $\mathrm{CC}$ and $\mathrm{NC}$ rates given in Table 1 are obtained assuming undistorted energy spectra above $5 \mathrm{MeV}$, which for transitions to active neutrinos has good empirical justification as mentioned above. We thank Prof. Mark Chen of SNO collaboration for communication on this point.

\section{References}

[1] The SNO Collaboration (Q.R. Ahmad et al.), (to appear in Phys. Rev. Lett.), nuclex/0204008.

[2] A. Bandyopadhyay, S. Choubey, S. Goswami and D.P. Roy, hep-ph/0203169, (to appear in Mod. Phys. Lett. A).

[3] See e.g. V. Barger, D. Marfatia and K. Whisnant, Phys. Rev. Lett. 88, 011302 (2002).

[4] J.N. Bahcall, M.H. Pinsonneault and S. Basu, Astrophys. J. 555, 990 (2001).

[5] F.L. Villante, G. Fiorentini and E. Lisi, Phys. Rev. D59, 013006 (1999).

[6] G.L. Fogli, E. Lisi, D. Montanino and A. Palazzo, Phys. Rev. D64, 093007 (2001).

[7] S. Fukuda et al., Super-Kamiokande collaboration, Phys. Rev. Lett. 86, 5651 (2001).

[8] The SNO Collaboration (Q.R. Ahmad et al.), Phys. Rev. Lett. 87, 071301 (2001)

[9] The SNO Collaboration (Q.R. Ahmad et al.), (to appear in Phys. Rev. Lett.), nuclex/0204009.

[10] S. Goswami, D. Majumdar, A. Raychaudhuri, Phys. Rev. D63, 013003 (2001); ibid hep-ph/9909453. S. Choubey, S. Goswami, N. Gupta and D.P. Roy, Phys. Rev. D64, $053002(2001)$;

[11] A. Bandyopadhyay, S. Choubey, S. Goswami and K. Kar, Phys. Lett. B519, 83 (2001); S. Choubey, S. Goswami, K. Kar, H.M. Antia and S.M. Chitre, Phys. Rev. D64, 113001 (2001); S. Choubey, S. Goswami and D.P. Roy, Phys. Rev. D65, 073001 (2002); A. Bandyopadhyay, S. Choubey, S. Goswami and K. Kar, Phys. Rev. D65, 073031 (2002).

[12] G.L. Fogli, E. Lisi, D. Montanino, A. Palazzo, Phys. Rev. D64, 093007 (2001); J.N. Bahcall, M.C. Gonzalez-Garcia, C. Pana-Garay, JHEP 0108, 014 (2001); P.I. Krastev and A.Yu. Smirnov, e-Print Archive: hep-ph/0108177; M.V. Garzelli and C. Giunti, JHEP 0112, 017 (2001). 
[13] J. N. Abduratshitov et al., SAGE collaboration, astro-ph/0204245; W. Hampel et al., GALLEX collaboration, Phys. Lett. B447, 127 (1999); M. Altman et al., GNO collaboration, Phys. Lett. B490, 16 (2000); E. Belloti, talk at Gran Sasso National Laboratories, May 17, 2002; T. Kirsten, talk at Neutrino 2002, Munich.

[14] B. Cleveland et al., Ap. J. 496, 505 (1998),

[15] M.B. Smy, talk at noon2001, hep-ex/0202020.

[16] V. Barger, D. Marfatia, K. Whisnant and B.P. Wood, hep-ph/0204253.

[17] P. Creminelli, G. Signorelli and A. Strumia, hep-ph/0102234 (updated version 3). 


\begin{tabular}{|c|c|c|}
\hline experiment & $R$ & composition \\
\hline$G a$ & $0.553 \pm 0.034$ & $p p(55 \%), B e(25 \%), B(10 \%)$ \\
$C l$ & $0.337 \pm 0.030$ & $B(75 \%), B e(15 \%)$ \\
$S K$ & $0.465 \pm 0.014(0.363 \pm 0.014)$ & $B(100 \%)$ \\
$S N O(C C)$ & $0.349 \pm 0.021$ & $B(100 \%)$ \\
$S N O(N C)$ & $1.008 \pm 0.123$ & $B(100 \%)$ \\
\hline
\end{tabular}

Table 1: The observed solar neutrino rates relative to the $S S M$ predictions (BP2000) are shown along with their compositions for different experiments. For the $S K$ experiment the $\nu_{e}$ contribution to the rate $R$ is shown in parantheses assuming $\nu_{e} \rightarrow \nu_{a}$ transition. In the combined Ga rate we have included the latest data from SAGE and GNO.

\begin{tabular}{|c|c|c|c|c|c|}
\hline $\begin{array}{l}\text { Data } \\
\text { Used }\end{array}$ & $\begin{array}{l}\text { Nature of } \\
\text { Solution }\end{array}$ & $\begin{array}{l}\Delta m^{2} \\
\text { in } \mathrm{eV}^{2}\end{array}$ & $\tan ^{2} \theta$ & $\chi_{\min }^{2}$ & $\begin{array}{c}\text { Goodness } \\
\text { of fit }\end{array}$ \\
\hline $\mathrm{Ga}+$ & LMA & $9.66 \times 10^{-5}$ & 0.41 & 35.95 & $80.08 \%$ \\
\hline $\mathrm{SK} / \mathrm{NC}+$ & LO & $1.04 \times 10$ & & & $09 \%$ \\
\hline $\mathrm{CC} / \mathrm{NC}+$ & VO & $4.48 \times 10^{-10}$ & 0.99 & 54.25 & $13.84 \%$ \\
\hline SKspec & SMA & $6.66 \times 10^{-6}$ & $1.35 \times 10^{-3}$ & 67.06 & $1.41 \%$ \\
\hline $\mathrm{Cl}+\mathrm{Ga}+$ & LMA & $6.07 \times 10^{-5}$ & 0.41 & 40.57 & $65.99 \%$ \\
\hline $\mathrm{SK}+\mathrm{CC}+$ & LOW & $1.02 \times 10^{-7}$ & 0.60 & 50.62 & $26.14 \%$ \\
\hline $\mathrm{NC}+\mathrm{SKspec}$ & VO & $4.43 \times 10^{-10}$ & 1.1 & 56.11 & $12.39 \%$ \\
\hline$+f_{B}$ free & SMA & $5.05 \times 10^{-6}$ & $1.68 \times 10^{-3}$ & 70.97 & $0.81 \%$ \\
\hline
\end{tabular}

Table 2: The $\chi_{\min }^{2}$, the goodness of fit and the best-fit values of the oscillation parameters obtained for the analysis of the global solar neutrino data. 


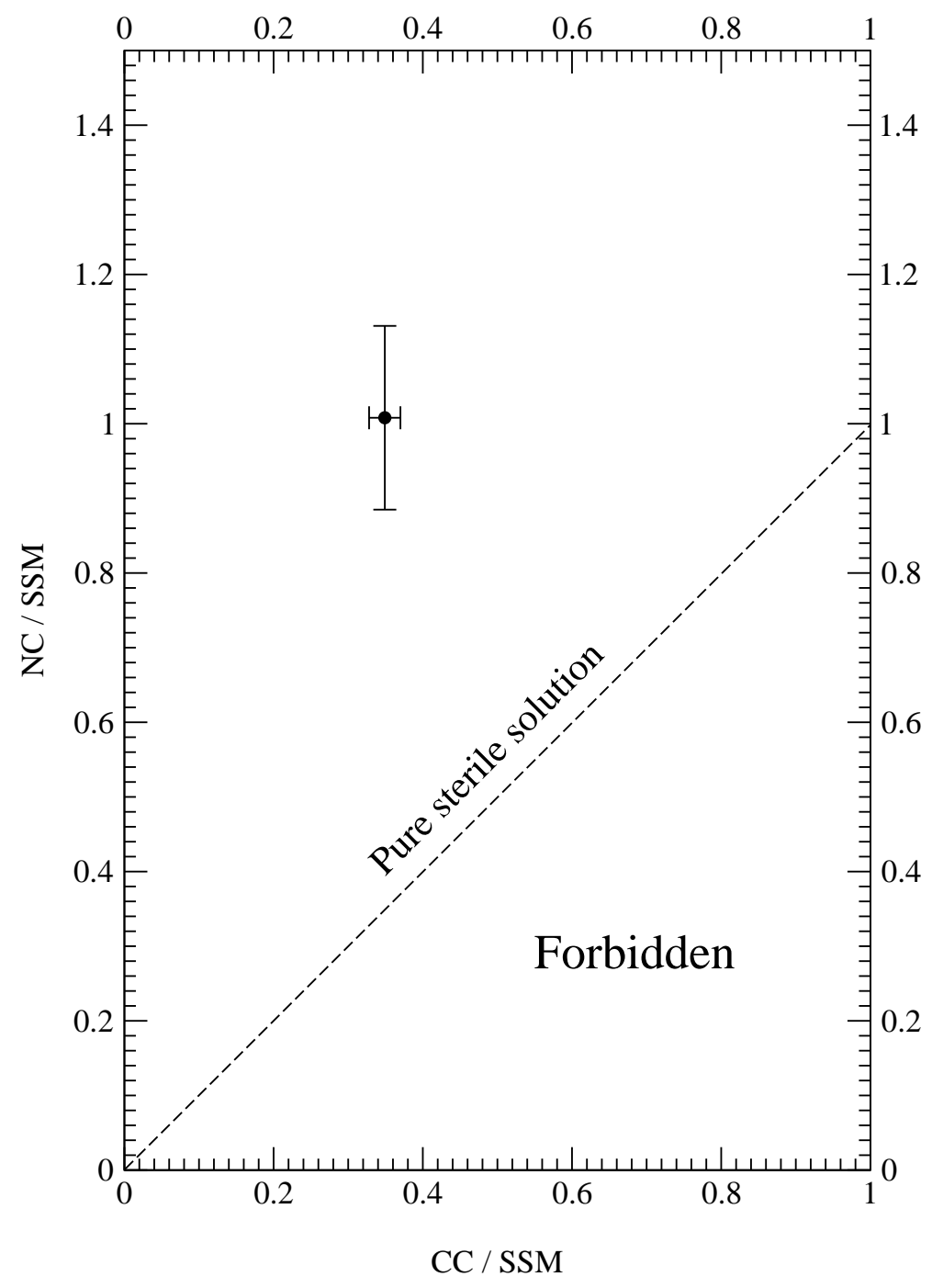

Figure 1: The $S N O C C$ and $N C$ rates shown relative to their $S S M$ predictions. The dashed line is the prediction of the pure $\nu_{e}$ to $\nu_{s}$ transition. The pure sterile solution is seen to be disfavored at $5.3 \sigma$. 


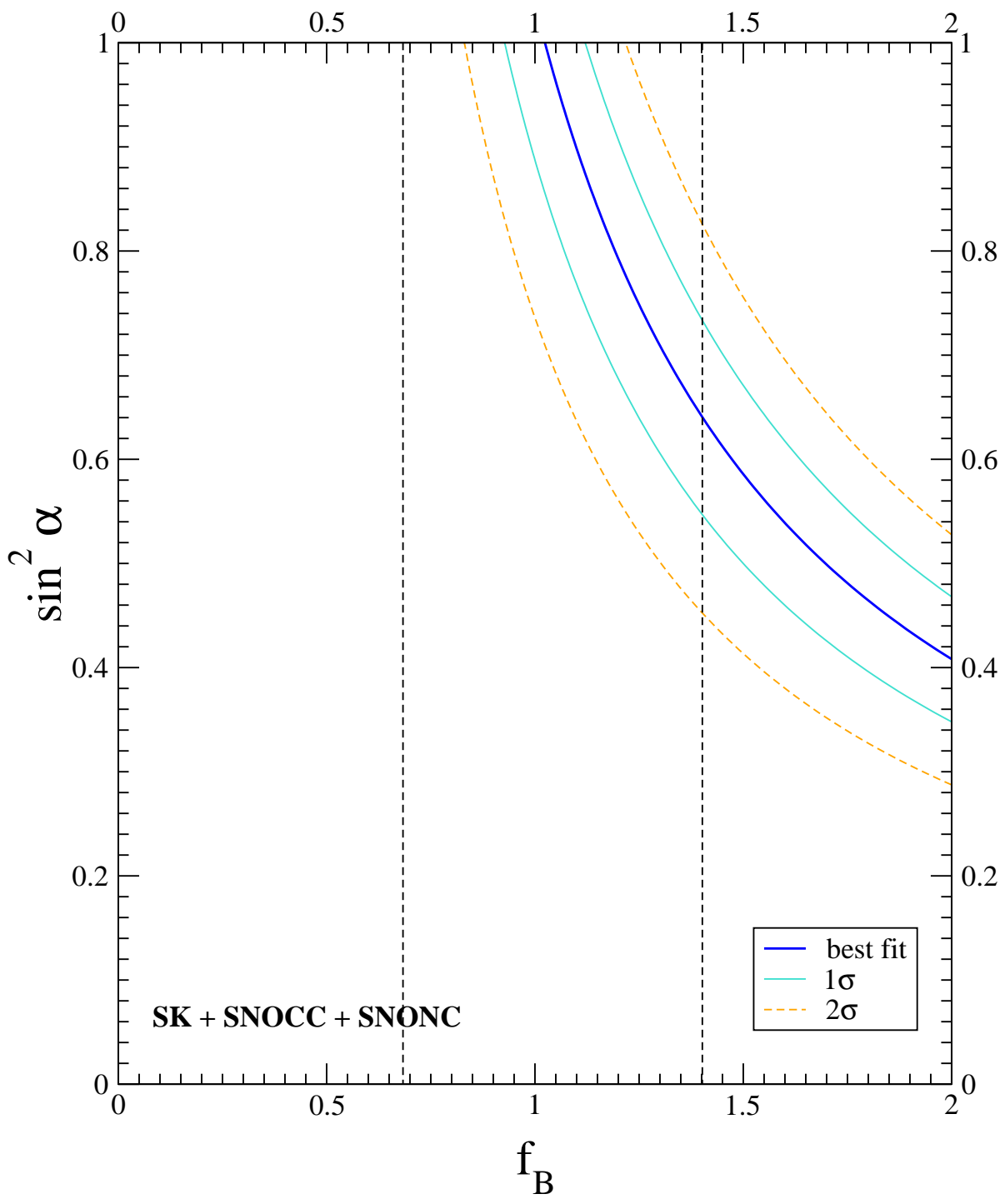

Figure 2: Best fit value of the ${ }^{8} B$ neutrino flux $f_{B}$ shown along with its $1 \sigma$ and $2 \sigma$ limits against the model parameter $\sin ^{2} \alpha$, representing $\nu_{e}$ transition into a mixed state $\left(\nu_{a} \sin \alpha+\right.$ $\nu_{s} \cos \alpha$ ). The dashed line denote the $\pm 2 \sigma$ limits of the $S S M$. 


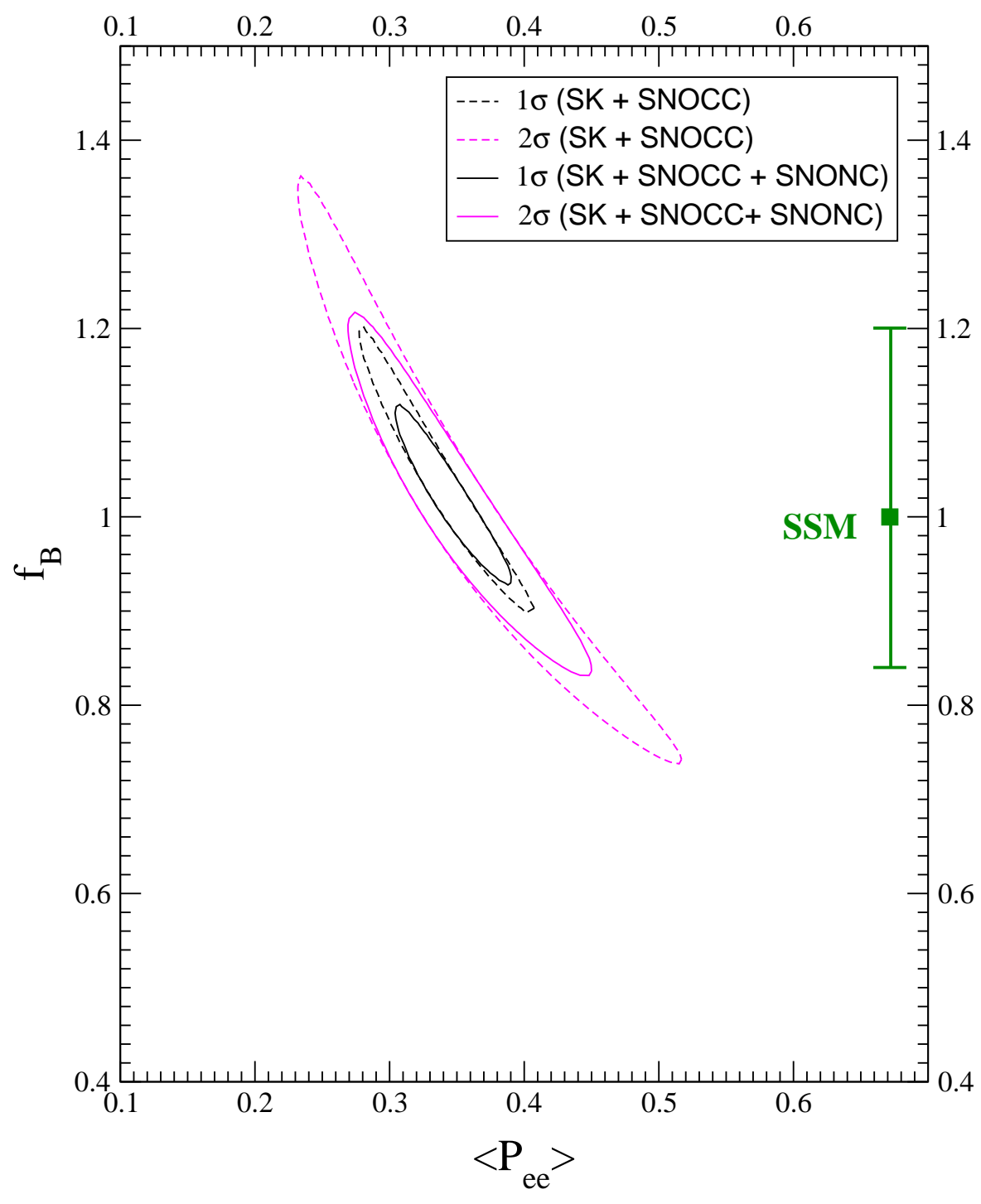

Figure 3: The $1 \sigma$ and $2 \sigma$ contours of solutions to the ${ }^{8} B$ neutrino flux $f_{B}$ and the $\nu_{e}$ survival probability $P_{e e}$ assuming $\nu_{e}$ to $\nu_{a}$ transition. The $1 \sigma S S M$ error bar for $f_{B}$ is indicated on the right. 


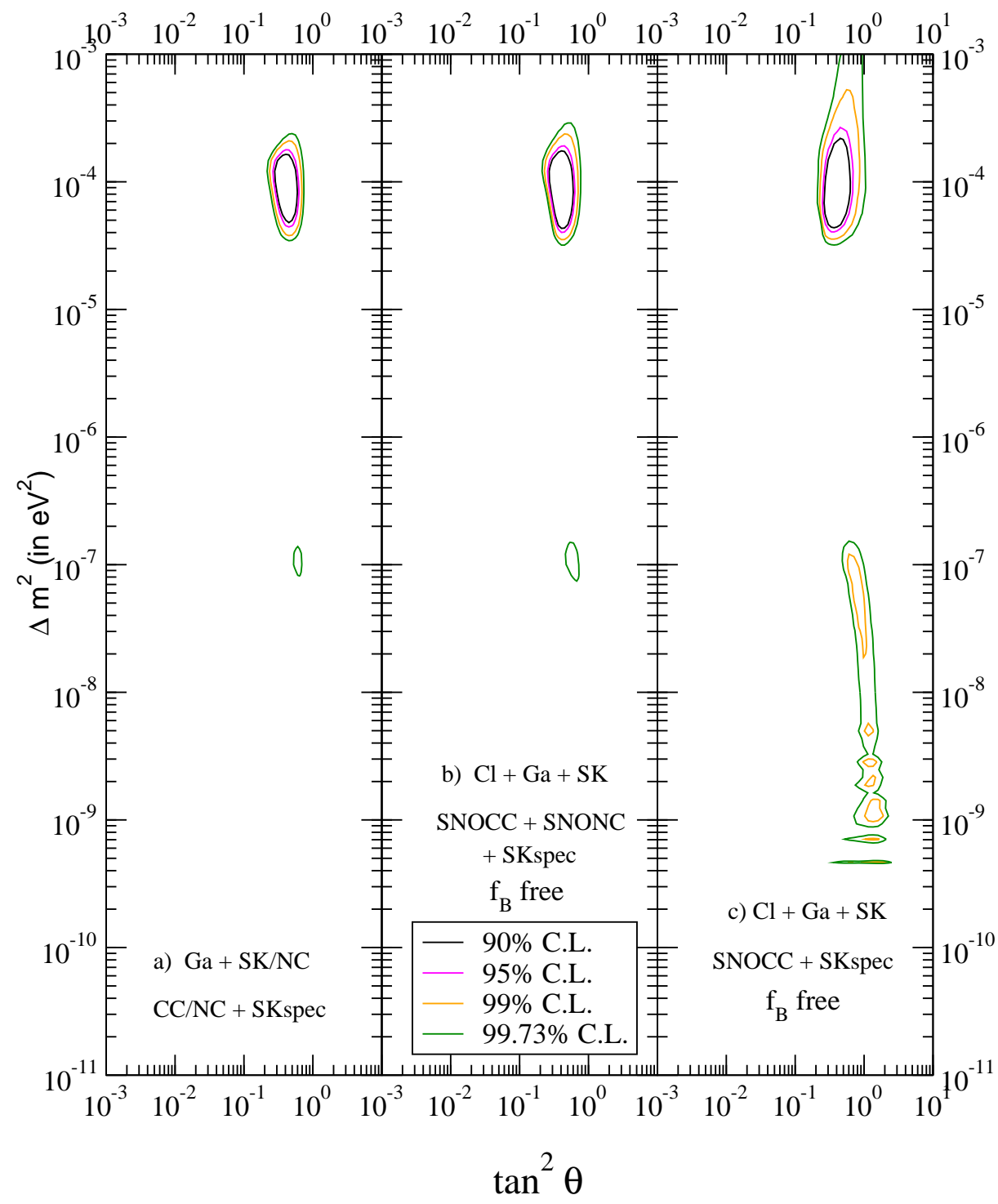

Figure 4: The $\nu_{e} \rightarrow \nu_{a}$ oscillation solutions to the global solar neutrino data using (a) Ga rate, the $S K$ zenith angle energy spectra and the $S K$ and $S N O(C C)$ rates, both normalised to the $S N O(N C)$ rate and (b) total $G a, C l, S K, S N O(C C)$ and $S N O(N C)$ rates along with the $S K$ zenith angle energy spectra, keeping the ${ }^{8} B$ flux normalisation $f_{B}$ free. In both cases we use the $S N O(N C)$ error as the error in the ${ }^{8} B$ flux. The case(c) is similar to (b), but without using the $\mathrm{SNO}(\mathrm{NC})$ rate. 